\title{
A bias error effect of sub-pixel edge location measurement by means of thermal camera
}

\author{
Robert Bąbka \\ Institute of Electronics and Control Systems, Częstochowa University of \\ Technology \\ Al. Armii Krajowej 17, 42-200 Częstochowa, Poland \\ e-mail: rbka@el.pcz.czest.pl
}

\section{POSTER SESSION}

Keywords: subpixel edge location, spatial resolution, optic transfer function, uncertainty of measurement, infrared radiation.

\section{Introduction}

Algorithms of the edge location with sub-pixel precision are usually considered in case of visual images analysis [1]. A similar solution can be applied to the edge analysis of the thermal objects registered by means of the infrared thermography technique [2].

There are three main problems of the edge location with sub-pixel precision:

1) errors of a method of radiation measurement [2],

2) finite spatial resolution of the thermal camera [2],

3) radiation distribution across the edge.

In visual systems, the third problem corresponds to irregular light distribution and causes bias in measurement of the edge location with sub-pixel precision. In case of the infrared measurement, radiation received from target is not only the reflected one, but mainly represents its own radiation. Therefore, the radiation distribution received from the edge neighbourhood depends on temperature and emissivity distribution.

The paper deals with a problem of influence of the temperature and emmisivity distribution to the bias in edge location. The paper contains:

1) infrared sub-pixel edge model analysis,

2) sub-pixel edge location algorithm description,

3) influence of the temperature and emmisivity distribution to the bias edge location analysis,

4) experimental results.

\section{Edge model}

An ideal physical edge may be considered as the step function determined in the spatial domain. In fact, edge images received from optical system are disturbed by lens aberration, disperse effects and detectors cross-talk. For visible optical systems as well as for infrared systems a degree of blurring is determined by Spread Response Function (SRF). In the one-dimensional case this function equals the Line Spread Function (LSF). Including meas- 
urement model of the thermal camera the edge related signal is described as fallows [2]:

$$
\text { (1) } \operatorname{EDGE}\left(x, \sigma, u_{0}, \Delta u\right)=u_{0}+\Delta u \cdot\left(1+\frac{\sqrt{2} E S F(x, \sigma)}{\sigma \sqrt{\pi}}\right) \text {, }
$$

where $R, B, F$ - calibration constants of a thermal camera, $u_{0}$ - lower radiation of the target, $\Delta u$ - radiation difference across the edge, $\sigma$ - parameter of LSF .

\section{Sub-pixel edge location algorithm}

Due to the lose of information caused by optical system filtering and spatial sampling, the method of sub-pixel edge location is based on interpolation algorithm. Interpolation reconstructs the edge signal (1) so the location of the edge can be estimated using equation:

$$
\frac{d^{2}}{d x^{2}}\left\{\operatorname{EDGE}\left(x_{e}, \sigma\right)\right\}=0 \text {, }
$$

\section{Bias error caused by a temperature distribution}

The figure 1 presents a problem with bias in case of exp-function-like temperature distribution in the edge neighbourhood. This distribution is the most likely as a solution of Fourier equation. The second derivate of interpolated edge signal is equal zero (2) in place different from real edge location. The bias effect is appeared (fig. 1d.)

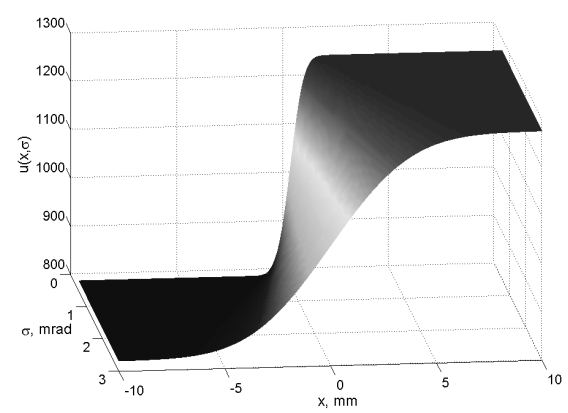

Fig. 1a. Non-disturbed edge model signal (equation 1)

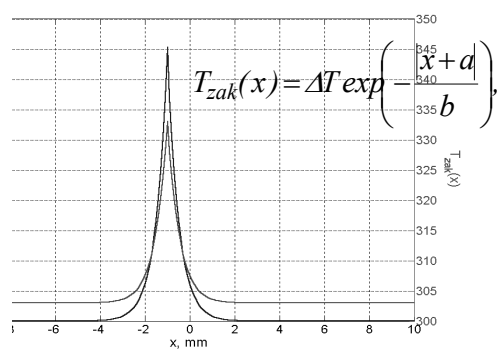

Fig. 1b. Temperature distribution as a source of the signal disturbance 

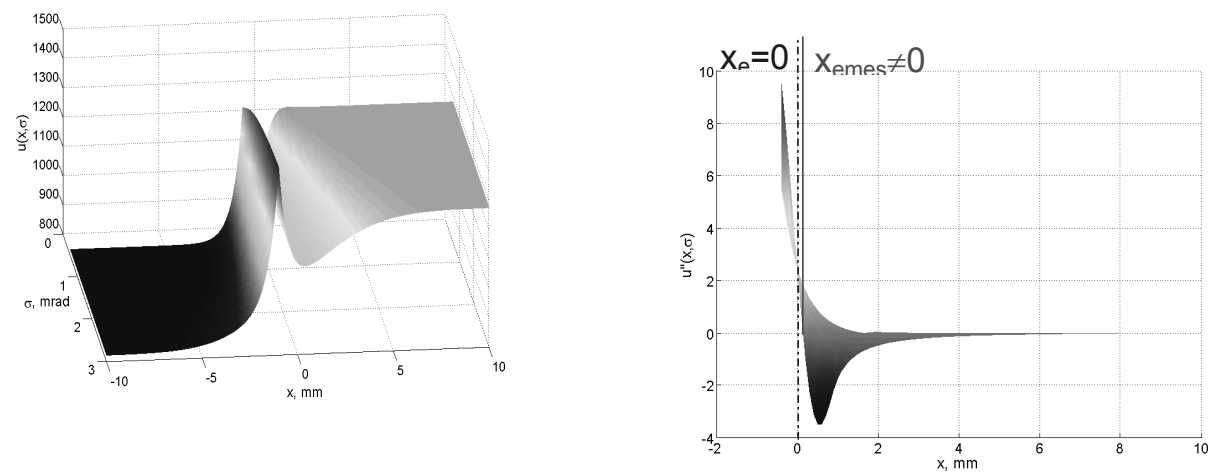

Fig. 1c. Disturbed edge signal (sum of 1a. and Fig. 1d. Bias error, according to (2) 1b.) measured edge location is at $x_{e m e s}$. Real position of the edge is at $x_{e}$

To prevent of bias author proposes the modification in sub-pixel edge location algorithm. The details will be presented in the paper.

\section{Experimental results and conclusions}

The laboratory stand for measurements was equipped with thermal camera ThermaCAM PM 595 (FLIR) and lenses $24^{\circ} \times 18^{\circ}$. As a thermal object a radiator $50 \times 50 \mathrm{~mm}( \pm 0.1 \mathrm{~mm})$ has been used. There were conducted 216 measurements collected at distances: 0.5, 1 and 2 meters. The standard deviation of measurements did not exceed 0.02 of pixel. Observed bias was as a resultant of optic distortion of lens and the exp-function-like temperature distribution effect. It did not exceed 0.5 of pixel. It means, in point of view of the uncertainty theory, bias can be treated as additional expected value of error to the result of measurement. This value is significant and cannot be neglected. In other hand, it can be predicted using appropriate model with modified parameters during calibration process.

\section{References}

[1] Boon G.W Shan Y.: Sub-Pixel Location of Edges With Non-Uniform Blurring: A Finite Closed-Form Approach. Image and Vision Computing, 18(2000) p. 1015-1023.

[2] Bąbka R., Minkina W., Wild W.: Evaluation of Mean Square Measure Application to Edge Detection of Objects in Thermal Images, Pomiary Automatyka Kontrola (PAK) 68(2001) №11p. 9-12 (in polish). 